\title{
High Power Cryogenic Targets
}

\section{Greg Smith}

Received: date / Accepted: date

\begin{abstract}
The development of high power cryogenic targets for use in parity violating electron scattering has been a crucial ingredient in the success of those experiments. As we chase the precision frontier, the demands and requirements for these targets have grown accordingly. We discuss the state of the art, and describe recent developments and strategies in the design of the next generation of these targets.
\end{abstract}

Keywords Cryogenic LH2 target

PACS $24.80 .+\mathrm{y} \cdot 25.30 . \mathrm{Bf} \cdot 29.25 . \mathrm{-t}$

\section{Introduction}

Electron scattering experiments aimed at electroweak physics via the measurement of parity violating observables are placing greater and greater demands on cryogenic target development. Thick $\mathrm{LH}_{2}$ targets up to $150 \mathrm{~cm}$ long, and beam currents approaching nearly $200 \mu \mathrm{A}$ are being employed. The power associated with the ionization energy loss of the electron beam through these targets is approaching $5 \mathrm{~kW}$. At the same time these parameters are being pushed to such extreme frontiers, the allowable contribution of target noise to the measured asymmetry widths is being driven ever smaller, well below $100 \mathrm{ppm}$. Table 1 shows what has been achieved by some of the cutting edge targets developed to date, as well as goals for two future targets currently under development. We frame our discussion of target design around ongoing design work associated with one of these next generation targets, the Qweak target.

In considering the design of any given target, there are a number of knobs to turn to optimize that target's performance. These include the operating pressure and

This work was supported by Jefferson Science Associates, LLC under U.S. DOE Contract No. DE-AC05-06OR23177.

G. R. Smith

Jefferson Lab

Tel.: +757-269-5405

Fax: +757-269-5235

E-mail: smithg@jlab.org 
temperature, the flow velocity across the beam axis, the frequency and area over which the electron beam is rastered (dithered) across the target, the intrinsic diameter and the helicity reversal frequency of the electron beam, the design and thickness of the entrance and exit windows of the target cell, and the basic design of the cell which can affect the thermodynamic properties of the LH2 in the beam interaction volume, in particular. A number of factors constrain these design choices: the target length and beam current required to achieve the experiment's desired precision, the background generated from the target cell windows, safety concerns, the available cooling power, the pressure head of the target loop, the statistical width of the asymmetry differences measured in the experiment, the time available to design and build the target, as well as to perform the measurement, and finally, constraints associated with DOE 10CFR851, aka ASME compliance.

The Qweak target performance has been estimated based on that achieved for G0's target. The G0 target reported [2] a target width of $238 \mathrm{ppm}$. We scale the raster area, target length, and beam current for the two targets linearly. Although there is evidence from a number of sources that a target's performance scales faster than linearly with the massflow (G0 reported a cubic dependence), we scale this linearly as well since it clearly depends on cell design details. The G0 target had a LH2 flow longitudinal to the electron beam, whereas Qweak's target flow is transverse to the beam axis. Finally, recent studies at JLab have shown that the target width scales with the electron beam's helicity reversal frequency according to a power law with exponent 0.4 . Using the values in Table 1, a target width contribution of about $35 \mathrm{ppm}$ can be expected for the Qweak target based on these scaling arguments.

The Qweak target is the first to benefit from use of Computaional Fluid Dynamics (CFD) simulations for all aspects of its design. The focus of these efforts was on optimizing the design of the target cell in the beam interaction volume. However, aspects of the heater design, the heat exchanger, the windows, the dummy targets, as well as effects associated with the raster, and finally, various accident scenarios were also studied using this powerful new tool.

Table 1 Parameters of existing and future Cryogenic Targets

\begin{tabular}{lllllllll}
\hline Expt. & $\begin{array}{l}\mathrm{P} / \mathrm{T} / \dot{m} \\
\mathrm{psia} / \mathrm{K} / \mathrm{kg} / \mathrm{s}\end{array}$ & $\begin{array}{l}\mathrm{L} \\
\mathrm{cm}\end{array}$ & $\begin{array}{l}\mathrm{P} / \mathrm{I} / \mathrm{E} \\
\mathrm{W} / \mu \mathrm{A} / \mathrm{GeV}\end{array}$ & $\begin{array}{l}\mathrm{P}_{V} \\
\mathrm{~W} / \mathrm{cm}^{3}\end{array}$ & $\begin{array}{l}\mathrm{d}_{\text {beam }} \\
\mathrm{mm}\end{array}$ & $\begin{array}{l}\Delta \rho / \rho \\
\%\end{array}$ & $\begin{array}{l}\delta \rho / \rho \\
\mathrm{ppm}\end{array}$ & $\begin{array}{l}\text { Helicity } \\
\text { rev }(\mathrm{Hz})\end{array}$ \\
\hline Existing: & & & & & & & & \\
SAMPLE & $25 / 20 / 0.6$ & 40 & $700 / 40 / 0.2$ & 396 & 2 & 1 & $<1000$ & 60 \\
HAppEx & $26 / 19 / 0.1$ & 20 & $500 / 35-55 / 3$ & 76 & $5 \times 5$ & & 100 & 30 \\
PVA4 & $25 / 17 / 0.13$ & 10 & $250 / 20 / 0.85$ & 310 & 1.7 & 0.1 & 392 & 50 \\
E158 & $21 / 20 / 1.8$ & 150 & $700 / 12 / 48$ & 467 & 1 & 1.5 & $<65$ & 120 \\
G0 & $25 / 19 / 0.3$ & 20 & $500 / 40-60 / 3$ & 346 & $2 \times 2$ & $<1.5$ & 238 & 30 \\
\hline Future: & & & & & & & \\
Qweak & $35 / 20 / 1$ & 35 & $2500 / 180 / 1.1$ & 245 & $5 x 5$ & & $<4$ & 250 \\
MOLLER & & 150 & $5000 / 85 / 11$ & & & & $<26$ & 2000 \\
\hline
\end{tabular}




\section{Pressure \& Temperature}

A liquid Hydrogen target can operate over only a relatively narrow temperature range. Hydrogen freezes at around $14 \mathrm{~K}$, more or less independent of pressure. The temperature at which Hydrogen boils does depend on its pressure, varying from $22.2 \mathrm{~K}$ at 25 psia, to $25.2 \mathrm{~K}$ at 50 psia. Ideally, the target is sub-cooled several degrees below the boiling temperature in order to accomodate a small temperature rise in the beam interaction volume without boiling. The amount of sub-cooling is a compromise between this requirement, and the impact on the cooling power, which is proportional to the difference between the coolant supply temperature and the target operating temperature. In practice, most targets have an operating temperature of 19 or $20 \mathrm{~K}$.

There are also limitations on the operating pressure. A cryogenic target must never go sub-atmospheric $(<14.7$ psia) or the possibility of air leaking into the plumbing could lead to a catastrophe. Higher pressures have the disadvantage that they lead to thicker windows (higher backgrounds), higher warm gas storage pressures, and more gas inventory. However, higher pressures offer a number of advantages as well. Foremost among these is superior cavitation headroom. Cavitation can occur at the trailing edge of the pump blades if the pressure there drops below the vapor pressure. The vapor pressure of $19 \mathrm{~K} \mathrm{LH}_{2}$ is 9.9 psia (13.6 psia for $20 \mathrm{~K} \mathrm{LH}_{2}$ ). So raising the pressure of a $20 \mathrm{~K} \mathrm{LH}_{2}$ target from 25 psia to 35 psia doubles the cavitation headroom. Higher pressure also means a higher boiling temperature, which can be taken advantage of in one of two different ways. The target could be operated at higher temperature, which provides more cooling power. Or the target could be kept at a fixed temperature, in which case one accrues more sub-cooling.

We explored the potential benefit of the latter option in the context of film boiling at the windows of the target. Qualitatively, as the target sub-cools, the heat flux into the liquid across the film at the window improves, which helps lower the window temperature and thus mitigates the impact of film boiling at the window. To quantify this effect, we made use of a study described in [1]. There, the effect of sub-cooling on the heat transfer q across the film was characterized by $F_{\text {sub }}=\frac{q_{\text {subcooled }}}{q_{\text {saturation }}}$. They found

$$
\mathrm{F}_{\mathrm{sub}}=1.0+12.236\left[\frac{\left(\mathrm{C}_{\mathrm{p}} \rho \mathrm{k}\right)_{\mathrm{l}}^{0.5}(\Delta \rho)^{0.125}}{\lambda \rho_{\mathrm{v}}^{0.75} \sigma^{0.375}}\right](\Delta \mathrm{T})_{\mathrm{sub}}
$$

where $\mathrm{C}_{p}$ is the specific heat, $\rho$ the density, k the thermal conductivity, $\Delta \rho$ the liquidfilm density difference, $\lambda$ the heat of vaporization, and $\sigma$ the liquid surface tension. For a $19 \mathrm{~K} \mathrm{LH}_{2}$ target, $\mathrm{F}_{\text {sub }}$ rises from 1.18 to only 1.26 as the pressure rises from 25 psia to 45 psia. Thus, although this effect is real, it's too small to be a driver in target design. The bottom line is that most targets operate at around 25 psia. Qweak aims for 35 psia, mainly to achieve more cavitation headroom.

\section{Cooling Power}

The main factor driving the cooling power requirement for any given target is the ionization energy loss of the beam, which is given by $\mathrm{P}_{b}(\mathrm{~W})=\mathrm{I}_{b}(\mu \mathrm{A}) \rho\left(\mathrm{g} / \mathrm{cm}^{3}\right) \mathrm{t}(\mathrm{cm})$ $\mathrm{dE} / \mathrm{dx}\left(\mathrm{MeV} / \mathrm{g} / \mathrm{cm}^{2}\right)$. For the Qweak target, for example, $\mathrm{I}_{b}=180(\mu \mathrm{A}), \rho=0.072$ $\left(\mathrm{g} / \mathrm{cm}^{3}\right), \mathrm{t}=35(\mathrm{~cm})$, and $\mathrm{dE} / \mathrm{dx}=4.653\left(\mathrm{MeV} / \mathrm{g} / \mathrm{cm}^{2}\right)$, so $\mathrm{P}_{b}=2.1 \mathrm{~kW}$. Beyond this, heat must be available to take away in order to control the target's temperature 
via a heater. The pump motor puts heat into the liquid. There are of course conductive losses between the $20 \mathrm{~K}$ target and the $300 \mathrm{~K}$ outside world. Finally, viscous heating of the target loop can also occur: Viscous Heating $(\mathrm{W})=\frac{\text { Flow }(\mathrm{l} / \mathrm{s}) \mathrm{Head}(\mathrm{psi})}{\text { pump efficiency }} \times 6.89$. But since the head $\mathrm{h}_{\mathrm{L}}=\mathrm{f} \frac{\mathrm{L}}{\mathrm{d}} \frac{\mathrm{v}^{2}}{2 \mathrm{~g}}$, the viscous heating goes like $\mathrm{v}^{3}$ ! (Note that the pressure head $\left.\Delta \mathrm{P}=\mathrm{h}_{\mathrm{L}} \rho \mathrm{g}\right)$. Most targets do not operate in the head and flow regime where this is important. Clearly however, this can quickly get out of hand if not accounted for in the design. For the Qweak target, we require the viscous heating not exceed $10 \%$ of the total heat load. The effect of all these other cooling power contributions adds another $500 \mathrm{~W}$ to the $2.1 \mathrm{~kW}$ of beam power required for the Qweak target, for example.

\section{Elements of the Target Loop}

The $\mathrm{LH}_{2}$ is circulated in a closed loop. The principle elements of the loop are the cell where the beam interacts with the liquid, a heater, a pump, and a heat exchanger. The loop is usually plumbed to a ballast tank to store the gas when it is warm, and to provide a pressure reservoir when the target is cold. Temperature and pressure instrumentation are provided at various points. A motion mechanism allows the target to be positioned on the beam, as well as enabling the use of other solid targets for various purposes in the experiment.

\subsection{Cell Design}

CFD simulations can play a very important role in the design of the cell. For the Qweak target, both longitudinal and transverse designs were studied looking at factors including the average and maximum temperature rise and density reduction in the beam interaction volume, the heating at the windows, the effects of design changes on the head and Hydrogen volume. We found that for the design parameters of the Qweak target, a transverse design offered about a factor of two better performance in all these areas. The final design we arrived at is shown in Figure 1.

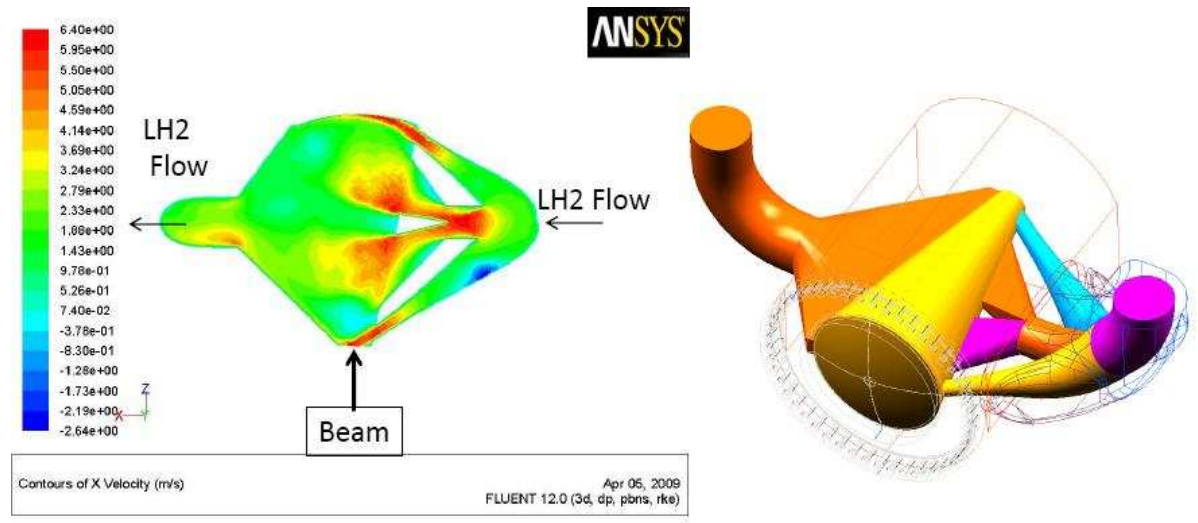

Fig. 1 Left: CFD simulation of the Qweak target cell, showing contours of velocity across the cell. Right: CAD model of the cell's flowspace. 


\subsection{Heat Exchanger}

In order to meet the challenges of providing $2.5 \mathrm{~kW}$ of cooling power, we developed a hybrid heat exchanger (HX) for Qweak's target. It combines the $1.2 \mathrm{~kW}$ cooling power of the JLab End Station Refrigerator (which supplies $15 \mathrm{~K}$ Helium coolant at 12 atm), with the excess capacity of the Central Helium Liquifier (4 K Helium coolant supplied at $3 \mathrm{~atm})$. Both coolant circuits feed a single counterflow HX wound with $1 / 2$ " Copper fin tube (16 fpi). The HX was designed analytically. CFD simulations were performed to check the head calculation, and to insure that Hydrogen ice would not form anywhere in the HX.

\subsection{Heater}

The heater for the Qweak target was designed analytically, and checked with CFD calculations. We settled on a short, four layer set of windings using 13 AWG Nichrome wire wound on a $1 / 16$ " thick G10 frame. Two coils connect in parallel to deliver 1.2 $\Omega$. The heater should not boil the liquid even at its design power of $2500 \mathrm{~W}$.

\subsection{Pump}

The pressure head and the mass flow (capacity) required are the dominant design factors for the pump. Traditionally, vane-axial pumps have been used to circulate the $\mathrm{LH}_{2}$ around the loop. For the higher head (1.3 psi) and higher capacities (15 liters/s) required of next generation targets like Qweak's, the specific speed dictates a centrifugal pump be used.

\section{Safety}

Three accident scenarios need to be thoroughly studied and accounted for in the design. The first of these is the relief calculation, which accounts for a sudden loss of insulating vacuum, and the subsequent rapid boiloff of the target liquid. The relief plumbing must be adequately sized to handle this kind of accident. The second scenario is the vent calculation, in which there is a rupture of the cryo-loop, presumably at the one of the thin windows. This puts $\mathrm{LH}_{2}$ into the scattering chamber. Vent plumbing on the scattering chamber must be designed to keep the system intact so that Hydrogen does not escape into the experimental hall. Finally, the accident scenario where Hydrogen does escape into the hall must also be examined.

Acknowledgements We acknowledge the contributions made by S. Covrig to the work reported here, in particular for the CFD calculations which he performed.

\section{References}

1. P.G. Brentari, "Boiling Heat Transfer for Oxygen, Nitrogen, Hydrogen, and Helium", NBS Technical Note 317, (1965).

2. S.D. Covrig, et al., "The Cryogenic Target for the G0 Target at Jefferson Lab", Nucl. Instum. Meth. A551, 218-235, (2005). 